\title{
In Segregation and Phase Separation In Multilayer Structures
}

\author{
C. Dorin, C. Wauchope ${ }^{1}$, J. Mirecki Millunchick, C. A. Pearson ${ }^{2}$, Y. Chen ${ }^{3}$, and B. G. Orr ${ }^{3}$ \\ Department of Materials Science and Engineering, University of Michigan, Ann Arbor, MI \\ 48109-2136 \\ ${ }^{1}$ Electron Microbeam Analysis Laboratory, University of Michigan, Ann Arbor, MI 481092136 \\ ${ }^{2}$ Department of Computer Science, Engineering Science and Physics, University of Michigan \\ Flint, Flint, MI 48502 \\ ${ }^{3}$ The Harrison M. Randall Laboratory, University of Michigan, Ann Arbor, MI 48109
}

In this paper we investigate the effect of In surface segregation on the microstructure of short period superlattices (SPS) in two different material systems with equivalent nominally lattice misfit: AlAs/InAs and GaAs/InAs. The growth of the AlAs/InAs SPS at T $=500^{\circ} \mathrm{C}$ proceeded via the layer-by-layer growth mode. The cross sectional transmission electron diffraction (XTEM) image for this structure shows that the film is homogeneous and the individual SPS layers are well-defined (figure 1a). The Scanning Tunneling Microscopy (STM) image of this sample shows only monolayer roughness (figure 1b). SPS AlAs/InAs structures grown at $\mathrm{T}=$ $520^{\circ} \mathrm{C}$ exhibit regular and periodic compositional modulations (CM) perpendicular to the growth direction as indicated by (002) dark field XTEM (figure 2a). The appearance of lateral CM is confirmed by high angle annular dark field images (HAADF). X-ray energy dispersive spectroscopy (XEDS) line profiles for representative samples were performed in order $\mathrm{b}$ calculate the amplitude and wavelength of the compositional modulated regions.

The modulation wavelength of these structures is $26 \mathrm{~nm}$ along the [1 $\overline{1} 0]$ direction and $17 \mathrm{~nm}$ along the [110] direction $\mathrm{nm}$. The corresponding STM image (figure 2b) shows a rough surface with periodic features with lateral dimensions on the same order as the composition modulation observed by XTEM.

Although Ga and $\mathrm{Al}$ have similar lattice constants, the microstructure of GaAs/InAs SPSs is significantly different. Uniform and homogeneous superlattice structures were not obtained in the $\mathrm{GaAs} / \mathrm{InAs}$ system for the examined temperature range of $475^{\circ} \mathrm{C} \leq \mathrm{T} \leq 510 \mathrm{C}^{\circ}$. Instead, lateral composition modulation with varying degrees of regularly was always observed. For example, at $\mathrm{T}=480^{\circ} \mathrm{C}$ the growth front roughens for the first 40 cycles of the SPS deposition after which $3 \mathrm{D}$ islands nucleate on the surface. TEM shows regular lateral composition modulation up to this critical thickness, above which the structure exhibits irregular phase separation (figure 3a). At $\mathrm{T}=510^{\circ} \mathrm{C}$, the growth mode is $3 \mathrm{D}$ from the onset of growth and the structure is phase separated across the entire thickness.(Fig. 3b).

We demonstrate that In segregation and roughening determine the microstructure ofthe deposited structure. High quality SPS structures can be achieved when the growth mode is layer by layer. Roughening and 3D island nucleation is correlated to phase separation in the lateral direction. We show that the growth mode can be controlled by tuning the mismatch between the SPS layers via In surface segregation. A kinetic model of In segregation demonstrates that the SPS layers are more intermixed for the AlAs/InAs structure over this temperature range. Thus, the lattice mismatch is lower in these structures and the driving force for roughening is reduced. 

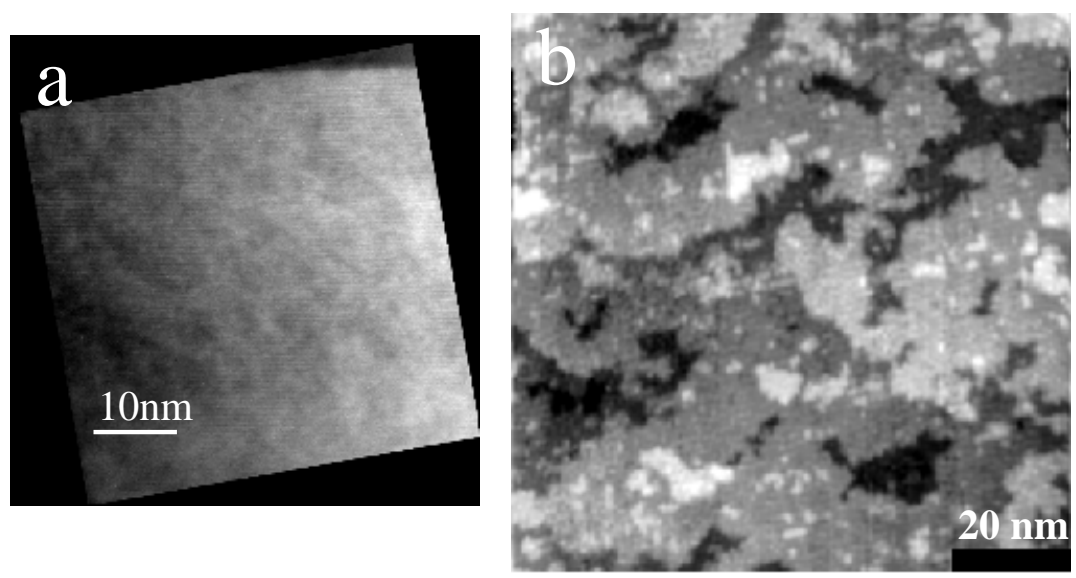

Fig. 1 Cross sectional [110] bright field TEM (a) and corresponding STM image (b) of an AlAs/InAs SPS grown at $\mathrm{T}=500^{\circ} \mathrm{C}$. The TEM image shows no evidence of lateral $\mathrm{CM}$ and the STM image shows large terraces characteristic to a smooth surface
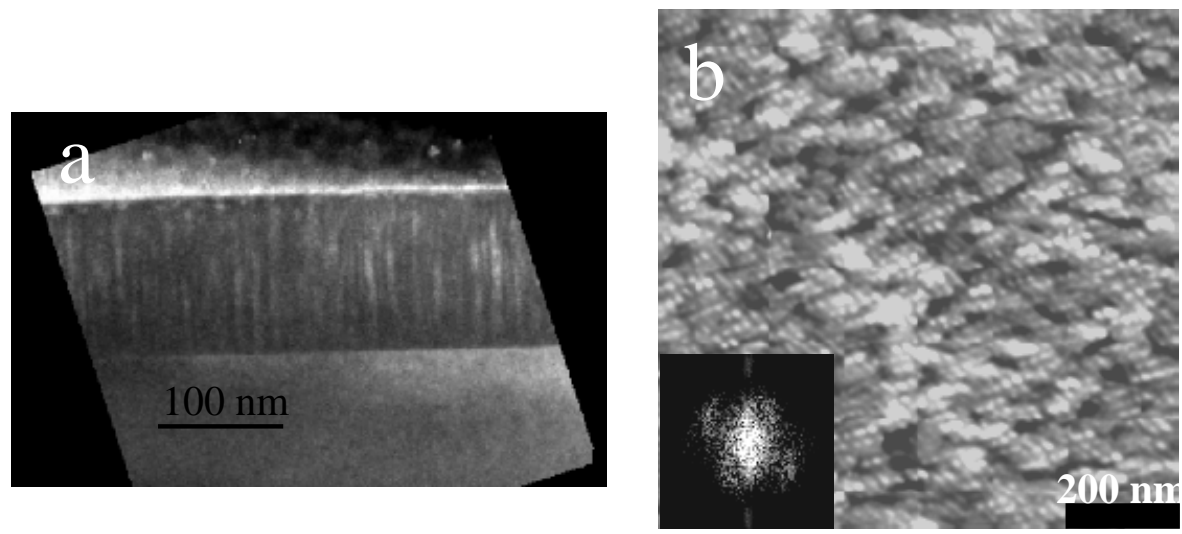

Fig. 2 Cross sectional 002 dark field TEM (a) and corresponding STM image (b) of an AlAs/InAs SPS grown at $\mathrm{T}=520^{\circ} \mathrm{C}$. The TEM image shows image contrast due to the presence of lateral CM and the STM image shows a rough surface with periodic features. The inset in $b$ is the FFT of the image. The lateral separation of features in the STM image is $27 \mathrm{~nm}$ in agreement with the modulation wavelength observed by XRD and XEDS.
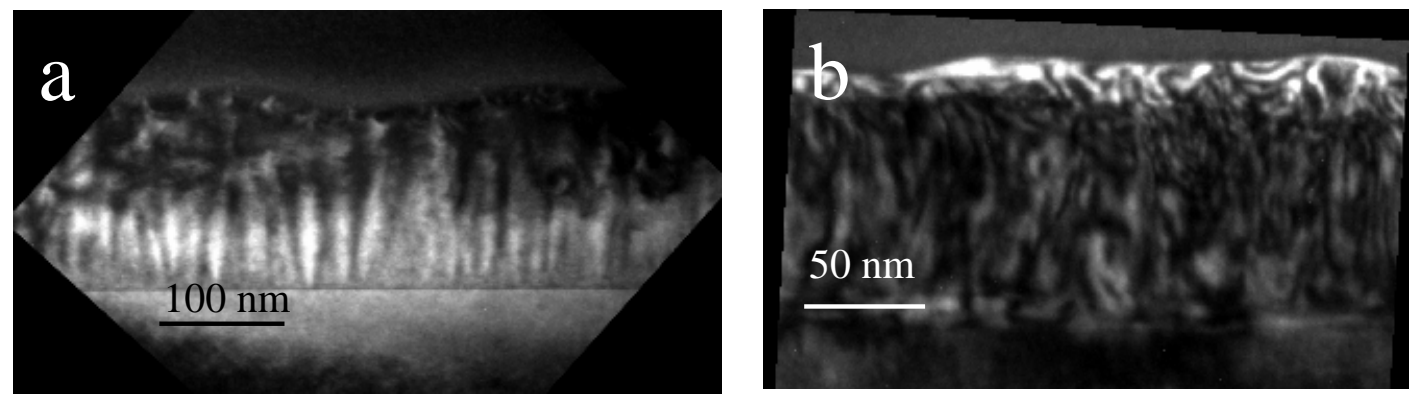

Fig. 3 Cross sectional TEM micrograph for the a) GaAs/InAs sample grown at $\mathrm{T}=480^{\circ} \mathrm{C}$ showing lateral $\mathrm{CM}$ below a certain thickness and b) GaAs/InAs sample grown $\mathfrak{a} \mathrm{T}=510^{\circ} \mathrm{C}$ showing less regular lateral CM and rough interfaces. 